\title{
Quel(s) rôle(s) pour l'enseignant.e et les élèves dans l'avancée du temps didactique en demi-fond : deux études de cas en primaire et au secondaire I à Genève
}

\author{
Bernard Poussin et Adrian Cordoba
}

Bernard Poussin.
Adrian Cordoba.

Résumé. Cette recherche s'inscrit dans un projet regroupant plusieurs instituts romands de formation des enseignant.es dans la perspective de construire des moyens d'enseignement innovants destinés aux enseignant.es. Cette étude concerne une enseignante du primaire et un enseignant d'éducation physique (EP) du secondaire I à propos de la mise à l'épreuve du terrain scolaire d'une forme de pratique scolaire (FPS) en demi-fond. La méthodologie s'inspire d'un dispositif de type ingénierie didactique et porte sur la manière dont l'enseignante et l'enseignant s'en emparent et font évoluer le temps didactique, en nous attachant également $\mathrm{au}(\mathrm{x})$ rôle(s) des élèves dans ce processus. Nous nous inscrivons dans le cadre théorique de l'action conjointe en didactique. Les résultats montrent que l'avancée du temps didactique dépend en grande partie de la compréhension que se font les enseignant.es volontaires des enjeux d'apprentissage de la FPS et notamment du rôle des observateur.trices, et nous observons des effets contrastés de leur épistémologie pratique selon les degrés scolaires.

Mots-clés. Demi-fond, Epistémologie pratique, Avancée du temps didactique, Rôles sociaux

\section{Introduction}

Cette communication s'inscrit dans un projet de recherche qui regroupe plusieurs instituts romands de formation des enseignant.es avec la perspective de construire des moyens d'enseignement innovants en éducation physique destinés aux enseignant.es. Avec un dispositif de type ingénierie didactique (Artigue, 2002), les formateur.es/chercheur.euses ont construit des formes des pratiques scolaires (FPS) en demi-fond adaptées à chaque cycle d'enseignement avec la volonté de respecter, d'une part, les prescriptions du plan d'études romand (PER) (Conférence intercantonale de l'instruction publique, 2010) et, d'autre part, les enjeux culturels de cette pratique (Lab, 2017). Ces FPS ont ensuite été proposées aux enseignant.es volontaires de cinq cantons romands pour les mettre à l'épreuve du terrain scolaire dans la perspective d'une validation interne par confrontation d'analyses a priori et a posteriori.

Pour la FPS de la fin du cycle 1 (élèves de 7-8 ans), la compétence attendue consiste à gérer sa vitesse de course pendant huit minutes pour courir sans interruption et parcourir la même distance pendant la première et la deuxième partie de la course, quatre minutes dans un sens puis quatre dans l'autre.

Pour la FPS de la fin du cycle 3 (élèves de 14-15 ans), la compétence attendue consiste à construire et gérer un projet de course en peloton constitué de coureurs de même potentiel aérobie pour respecter trois vitesses de course différentes en 15 minutes et établir une performance individuelle lors de la dernière vitesse de course. Les trois vitesses imposées (60, 85, 100 \% de la VMA) conservent le sens de l'entraînement sportif (Bergé, 2007) et se déroulent sur une piste fractionnée par des cônes disposés tous les 25 mètres, ce qui donne la vitesse de course sur des séquences de 1’30. Les critères d'évaluation concernent: (1) le respect des trois vitesses de course en peloton pour les deux premières et individuellement pour la dernière; (2) la prise de risque dans la construction du projet. Dans cette FPS, seules deux séquences de 1'30 de course sont imposées pour les deux premières vitesses (60 et $80 \%$ de la VMA), quatre séquences de course sont donc laissées au choix des différents groupes ; (3) la désolidarisation du peloton qui pénalise les groupes lorsque l'écart entre les coureur.euses est trop grand ; (4) la distance parcourue individuellement lors des deux dernières séquences de course. 
Quel(s) rôle(s) pour l'enseignant.e et les élèves dans l'avancée du temps didactique en...

Notre problématique porte sur la manière dont les enseignant.es s'emparent de ces FPS et font évoluer le temps didactique (Mercier, 2001) à travers les dispositifs et les régulations qu'ils proposent en nous attachant également $\mathrm{au}(\mathrm{x})$ rôle(s) des élèves dans ce processus. Dit autrement, s'il revient à l'enseignant l'exposition du savoir, son avancement reste cependant influencé par l'activité des élèves et le temps d'apprentissage. Pour appréhender ces interactions didactiques, nous nous inscrivons dans le cadre théorique de l'action conjointe en didactique (AmadeEscot \& Loquet, 2010). Nous tentons de comprendre le rôle de l'épistémologie pratique de l'enseignant.e et la manière dont elle influence ses choix didactiques lorsqu'il.elle se confronte à une proposition didactique innovante. Nous faisons référence ici aux théories implicites de l'enseignant.e composées de croyances, de valeurs, de savoirs experts qui l'amènent à choisir certaines tâches et à réguler en pointant certains éléments de l'activité en classe.

\section{Méthodologie}

Pour cette contribution, nous nous sommes focalisés sur l'expérimentation de cette FPS dans deux degrés scolaires du canton de Genève : Sophie, une enseignante titulaire d'une classe mixte de fin du cycle 1 ; Romain, un enseignant d'EP avec une classe de filles de fin du cycle 3. Du point de vue méthodologique, la recherche est organisée en quatre phases : (1) présentation de la FPS à partir d'un document qui précise les enjeux d'apprentissage ; (2) enregistrement vidéo des leçons ; (3) entretiens d'auto-confrontation en début de cycle d'enseignement (AC1) après les deux premières leçons et à la fin du cycle d'enseignement (AC2). Ces entretiens sont structurés par des extraits vidéo choisis par les chercheurs et sur lesquelles nous proposons aux enseignant.es de réagir Les données nous permettent d'identifier les rôles respectifs des élèves et de l'enseignant.e dans l'avancée du temps didactique à partir des descripteurs de l'action conjointe (Sensevy, 2007).

\section{Résultats et discussion}

\section{Le cas de Sophie}

Lors de la deuxième leçon d'un cycle de cinq leçons, Sophie propose, après avoir animé un échauffement articulaire, une situation nommée «le serpent» dans laquelle les élèves par groupe doivent suivre l'élève qui se trouve en tête de colonne. Lors du premier entretien, l'enseignante affirme que cet exercice devait permettre aux élèves de se rendre compte que chacun.e doit trouver son propre rythme et qu'il est difficile de suivre quelqu'un qui ne court pas à la même vitesse que soi.

Puis, Sophie propose aux élèves de courir pendant huit minutes sur un parcours délimité par des fanions. La classe est séparée en deux groupes distincts, des coureur.euse.s et des observateur.trices qui comptabilisent le nombre de tours effectué par leur binôme. Pendant cette situation, Sophie s'attache à rendre les élèves attentifs à ne pas partir trop vite, à bien respirer.

Après avoir ramassé les papiers sur lesquels les observateur.trices ont comptabilisé les tours réalisés par leurs camarades, Sophie revient avec l'ensemble des élèves sur ce qui lui semble essentiel pour réussir dans cette FPS en adoptant une posture topogénétique de surplomb :

- Sophie : « ... certains ont des difficultés avec le souffle (...) certains n’ont pas trouvé son propre rythme, et il $\mathrm{y}$ en a qui commencent très, très vite».

Lors de cette étape, Sophie sans se préoccuper des résultats de l'observation, expose les moyens à mettre en œuvre devant permettre aux élèves de construire les compétences propres à cette FPS : une technique respiratoire sur laquelle les élèves doivent s'appuyer pour trouver leur propre rythme, une allure de départ conforme à leur potentiel aérobie. Ces propos sont confirmés pendant l'entretien : 
Actes de la 11ème Biennale de l'ARIS : Former des citoyens physiquement éduqués. Axe 2 - Vers une éducation physique de qualité, 10.25518/sepaps20.446

- Sophie (AC1) : « ...je trouve qu'il faut leur faire prendre conscience, que l'intérêt n'est pas de leur faire courir le plus de tours mais (...) mais courir régulièrement... ». (...) S'il est trop fatigué c'est parce qu'il a trop forcé, il n’a pas bien respiré».

Pendant l'auto-confrontation, l'enseignante se rend également compte grâce aux extraits visionnés et aux relances du chercheur que les situations qu'elle a proposées ne permettent pas aux élèves d'identifier leur propre allure. Cette position est partagée par les chercheurs. En effet, les indicateurs que les observateur.trices doivent relever pendant la course ne donnent pas d'information sur la gestion de l'allure de course. De plus les injonctions qu'elle communique aux élèves en fin de leçon ne leur permettent pas de se transformer.

Ainsi, lors de la troisième leçon, après un échauffement articulaire, Sophie propose deux situations. Dans la première, il s'agit de comptabiliser le nombre de piquets franchis en trente secondes pendant huit répétitions, ce qui doit permettre aux élèves de construire une allure de course qu'ils.elles pourront maintenir pendant la FPS. Dans la deuxième situation, il s'agit pour les élèves de courir quatre minutes en enchainant des séquences de trente secondes et de maintenir le même nombre de piquets avec l'aide d'observateur.trices.

- Sophie : «...regardez le compteur des poteaux chaque 30", par exemple 6, et après les 30" suivantes, vous devez en faire 6, et pas 7 ou 8.

Après cette tâche, elle rassemble les élèves au centre de la salle et tente de réguler la vitesse de course de certains élèves :

- Sophie : «Charles a compté que les trois premiers tours il a fait neuf poteaux mais qu'après il était fatigué et qu'il a dû s'arrêter. D'après ton expérience, qu'est-ce que tu as appris, est-ce que neuf poteaux c'est bien, peut être tu devrais diminuer le rythme pour tenir les minutes, par exemple faire sept».

Nous constatons que les rétroactions du milieu ainsi que le rôle des observateur.trices en comptabilisant le nombre de poteaux franchis par les coureur.euses leur ont permis d'identifier une allure de course régulière adaptée à leur possibilité et de l'expérimenter.

Lors de l'entretien final, on retrouve également des traces du déplacement réalisé par Sophie dans sa manière de penser l'enseignement du demi-fond. Elle affirme que cela lui a permis de réfléchir à sa pratique et de prendre conscience qu'il ne suffit pas de faire courir les élèves pour qu'ils progressent mais que cela nécessite de préparer ces leçons avec rigueur en ciblant les enjeux d'apprentissage.

\section{Le cas de Romain}

Dans un premier temps, nous évoquons la troisième leçon d'un cycle de sept leçons. Pendant les deux premières leçons, les élèves ont réalisé : (1) un test pour déterminer leur VMA et constituer des groupes homogènes ; (2) la FPS. Cette $3^{\mathrm{e}}$ leçon, comme toutes les leçons qui suivront, commence par un échauffement cardio vasculaire et articulaire suivi par des exercices d'école de course. Après l'échauffement, Romain revient sur les résultats des élèves obtenus lors de la situation de référence pour leur faire constater que la plupart des groupes sont en difficulté pour respecter les différentes vitesses imposées. Romain propose alors aux filles trois séries de courses individuelles dans lesquelles il s'agit de respecter les différentes vitesses (figure 1). 
Quel(s) rôle(s) pour l'enseignant.e et les élèves dans l'avancée du temps didactique en...

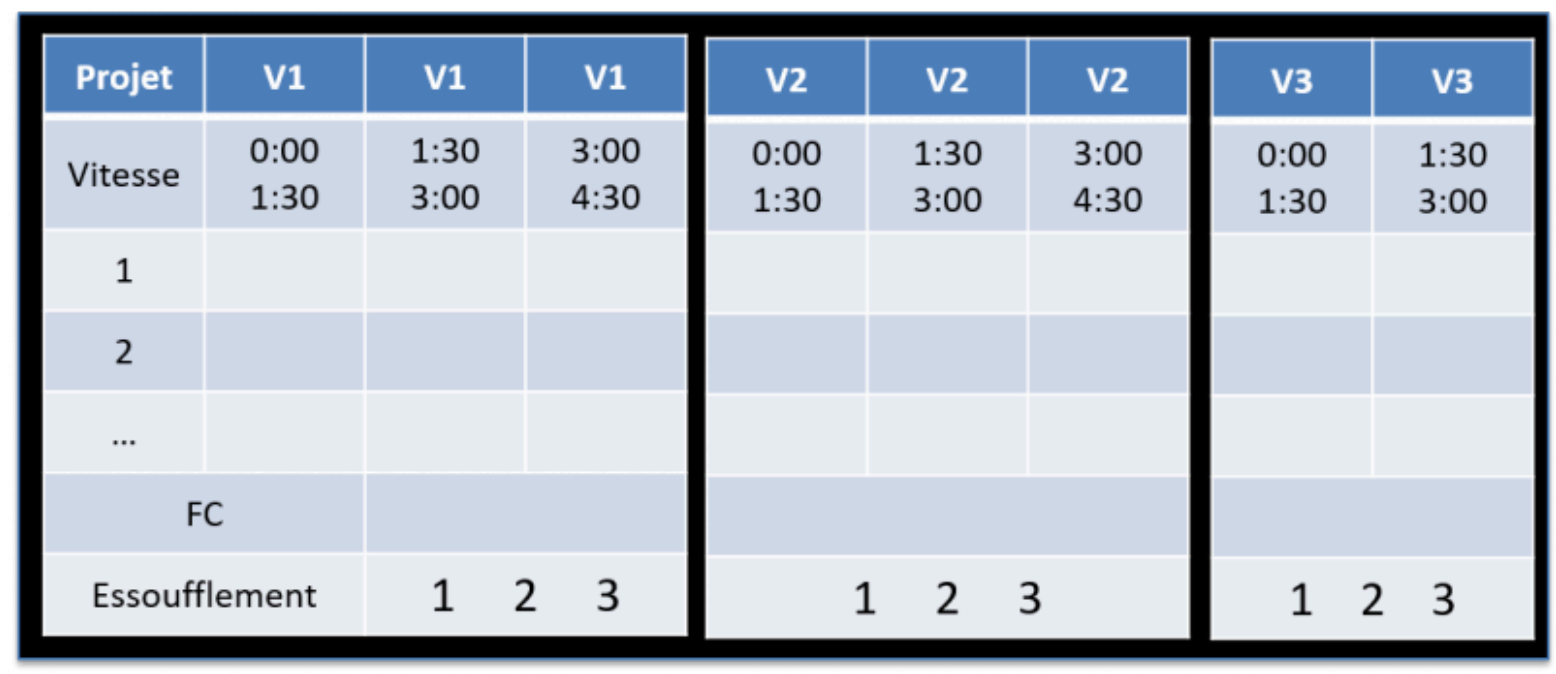

Figure 1 : Fiche d'observation individuelle des trois séries aux différentes vitesses

Nous notons que Romain reprend l'architecture de la fiche d'observation qui lui a été proposée avec la FPS mais en intégrant des indicateurs sur les sensations que les coureuses doivent relever après les séries de course. Lors de l'auto-confrontation, Romain nous indique que cette fiche est un outil pour informer les coureuses sur le respect des différentes vitesses et leur permettre de mettre en relation leur sensations et les vitesses de course. Son analyse a priori sur sa préparation atteste ces propos. Romain nous explique également que pour permettre aux élèves de progresser, il a l'intention d'engager les élèves dans une démarche réflexive à partir de la dévolution du problème suivant : Comment savez-vous que vous êtes à la bonne vitesse? Les temps de débat entre les séries ont permis aux élèves d'évoquer certaines règles d'action que Romain a progressivement institutionnalisées. Nous observons que Romain respecte les prescriptions du PER qui engagent les enseignant.es à intégrer cette capacité transversale dans leur démarche d'enseignement.

Pour cette situation les élèves sont répartis en binôme. L'enseignant se charge de donner le départ et de siffler les séquences de 1'30. Dès la première série, les observatrices s'adressent à leur partenaire pour tenter de réguler leur allure de course et leur permettre de franchir le nombre de cônes exigés :

- Elève 1 : «Ralentis ! Ralentis ! Nina, tu vas trop vite !».

- Elève 2 : « Tu es trop rapide ! Arrête-toi ! ».

Nous observons que ces interventions entraînent des changements brusques d'allure qui empêchent les coureuses de construire les règles d'action nécessaires aux respects des différentes vitesses. Romain s'en rend également compte et interpelle alors les coureuses pour leur rappeler une contrainte décisive de sa situation qui figure sur sa planification :

- Romain : «Je vous rappelle interdiction de marcher ou de vous arrêter ».

Il s'adresse également aux observatrices pour leur faire remarquer les effets de leurs communications.

- Romain : «Ralentis ! Ralentis ! Et après regardez, elles accélèrent ».

Nous estimons que ces régulations sont réalisées dans la perspective de maintenir la relation didactique (Amade-Escot, Verscheure \& Devos, 2002) et permettre aux élèves de se confronter aux enjeux d'apprentissage de cette FPS. 
Actes de la 11ème Biennale de l'ARIS : Former des citoyens physiquement éduqués. Axe 2 - Vers une éducation physique de qualité, 10.25518/sepaps20.446

Pendant l'auto-confrontation, lorsque le chercheur l'interroge sur cet extrait, Romain nous informe sur le rôle que les observatrices devraient tenir :

- Romain (AC) : «Les observatrices doivent informer les coureuses sur la vitesse, le retard ou l'avance ». [...] C'est en gérant l'avancée du temps qu'elles devront donner des informations. [...] Idéalement, j'aimerais qu'elles leur disent dès le début qu'elles doivent aller moins vite. Et pas 10" avant le coup de sifflet ».

Nous constatons que Romain s'attend à ce que les observatrices relèvent non seulement des distances de course pour les communiquer aux coureuses en fin de course mais qu'elles soient également capables de coacher les coureuses en leur donnant des informations pendant la course.

Lors de la $7^{\mathrm{e}}$ et dernière leçon du cycle d'enseignement, par groupe de VMA, les élèves doivent rédiger leur projet puis elles se déplacent sur le stade pour réaliser la FPS. Dès la première séquence de 1'30, les coureuses des différents groupes sollicitent les observatrices pour recevoir des informations :

- Les coureuses : « Encore combien de cônes ? ». « On en est où ?».

- Les observatrices : «Ralentissez, encore un cône !».

On assiste à nouveau à des ralentissements fréquents à l'approche de la fin de la séquence voire des arrêts ou, à l'inverse, des accélérations pour franchir le nombre de cônes exigés. Romain intervient rapidement auprès des observatrices et des coureuses pour gommer ces comportements :

- Romain aux observatrices : «Pendant qu'elles courent ne leur dites rien ! ».

- Romain aux coureuses : «Ne changez pas votre vitesse»

Cet extrait nous confirme que Romain n'a pas permis aux observatrices de construire les compétences pour coacher de manière pertinente les coureuses. Nous estimons que la construction du rôle de coach nécessite l'introduction d'un contrat didactique particulier bien qu'au service d'un même projet. Lors de l'entretien final, il s'interroge encore sur le rôle que doit tenir les observatrices : entre ne donner aucune communication pendant la course ou les autoriser une fois la 1'30 terminée. Pour l'avenir, ces alternatives lui semblent importantes pour forcer les coureuses à moins se reposer sur les observatrices.

\section{Conclusion}

Cette recherche a permis de pointer l'importance d'articuler un milieu avec des indicateurs relevés par des observateur.trices pour autant de spécifier leur rôle. Les résultats chiffrés s'avèrent essentiels, d'une part, pour permettre aux élèves de revenir sur leur activité et construire un projet d'action leur permettant d'obtenir une performance. D'autre part, ils constituent dès lors un moyen pour l'enseignant.e de faire avancer le temps didactique. Bien que nous n'avons pas pu ici développer en détail les entretiens d'auto-confrontation, soulignons cependant leur apport dans la réflexion réalisée par les enseignant.es sur leur action et dans le questionnement/déplacement dans leur manière de penser l'enseignement et l'apprentissage en demi-fond.

\section{Bibliographie}

Amade-Escot, C., Verscheure, I., \& Devos, O. (2002). Milieu didactique et régulations comme outil d'analyse de l'activité du professeur en éducation physique. Les dossiers des sciences de l'éducation, 8, 87-97.

Amade-Escot, C., \& Loquet, M. (2010). L'approche didactique en EPS et en APSA : analyse de l'action conjointe en contextes d'intervention. In M. Musard, M. Loquet \& G. Carlier (Eds.), Sciences de l'intervention en EPS et en sport (pp. 
Quel(s) rôle(s) pour l'enseignant.e et les élèves dans l'avancée du temps didactique en...

185-207). Paris : Éditions EP\&S.

Artigue, M. (2002). Ingénierie didactique : quel rôle dans la recherche didactique aujourd'hui ? Les Dossiers des sciences de l'éducation, 8, 50-72.

Bergé, A. (2007). Une forme de pratique scolaire de course de durée. Les cahiers du CEDRE, 7, 53-59.

Conférence intercantonale de l'instruction publique de la suisse romande et du Tessin (2010). Plan d'études romand. Neuchâtel : CIIP.

Lab, F. (2017). Comment « réhabiliter » le demi-fond pour motiver les élèves à courir ? Baldi : Éditions universitaires européennes.

Mercier, A. (2011). Contribution d’Alain Mercier. Éducation et didactique, 5(2). 151-156.

Sensevy, G. (2007). Des catégories pour décrire et comprendre l'action didactique. In G. Sensevy \& A. Mercier (Eds.), Agir ensemble. L'action didactique conjointe du professeur et des élèves (pp. 13-49). Rennes : Presses Universitaires de Rennes.

PDF automatiquement généré le 2023-04-26 12:23:48

Url de l'article : https://popups.uliege.be/sepaps20/index.php?id=446

Publié par ULiège Library en Open Access et distribué suivant les termes et les conditions de la licence CC-BY (https://creativecommons.org/licenses/by/4.0/deed.fr) 